Volume 7, Number 9, SePTEMBer 2007

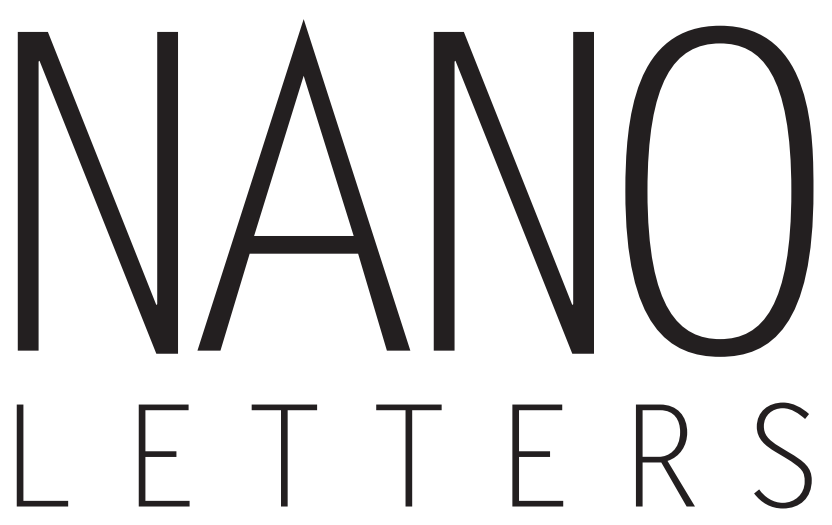

(C) Copyright 2007 by the American Chemical Society

\title{
Atomic-Scale Imaging in Real and Energy Space Developed in Ultrafast Electron Microscopy
}

\author{
Hyun Soon Park, J. Spencer Baskin, Oh-Hoon Kwon, and Ahmed H. Zewail ${ }^{\star}$ \\ Physical Biology Center for Ultrafast Science and Technology, Arthur Amos Noyes \\ Laboratory of Chemical Physics, California Institute of Technology, \\ Pasadena, California 91125
}

Received June 7, 2007

\begin{abstract}
In this contribution, we report the development of ultrafast electron microscopy (UEM) with atomic-scale real-, energy-, and Fourier-space resolutions. This second-generation UEM provides images, diffraction patterns, and electron energy spectra, and here we demonstrate its potential with applications for nanostructured materials and organometallic crystals. We clearly resolve the separation between atoms in the direct images and the Bragg spots/Debye-Scherrer rings in diffraction and obtain the electronic structure and elemental energies in the electron energy loss spectra (EELS) and energy filtered transmission electron microscopy (EFTEM).
\end{abstract}

The development of four-dimensional (4D) ultrafast electron microscopy and diffraction has made possible the study of structural dynamics with atomic-scale spatial resolution (so far in diffraction) and ultrashort time resolution. ${ }^{1}$ The scope of applications is wide-ranging, with studies spanning diffraction of isolated structures in reactions (gas phase), nanostructures of surfaces and interfaces (crystallography), and imaging of biological cells and materials undergoing first-order phase transitions. ${ }^{2-12}$ Typically, for microscopy the electron was accelerated to $120 \mathrm{keV}$ and for diffraction to $30 \mathrm{keV}$, respectively, and we had to address the issues of group velocity mismatch, in situ clocking (time zero) of the change, and frame referencing. ${ }^{1,3}$ One powerful concept implemented is that of "tilted pulses", which allow for the optimum resolution to be reached at the specimen. ${ }^{13}$

\footnotetext{
* Corresponding author. E-mail: zewail@ caltech.edu.
}

For ultrafast electron microscopy (UEM), the concept of "single-electron" imaging is fundamental. ${ }^{14}$ The electron packets, which have a well-defined picometer-scale de Broglie wavelength, are generated in the microscope $\mathrm{e}^{15}$ by femtosecond optical pulses (photoelectric effect) and synchronized with other optical pulses to initiate the change in a temperature jump or electronic excitation. Because the number of electrons in each packet is one or a few, the Coulomb repulsion (space charge) between electrons is eliminated and the temporal resolution reaches the ultimate, that of the optical pulse. The excess energy above the work function determines the electron energy spread and this can be minimized by tuning the photon energy. The spatial resolution is then only dependent on the total number of electrons because for each packet the electron "interferes with itself" and a coherent buildup of the image is achievable. 
The coherence volume, given by ${ }^{14}$

$$
V_{\mathrm{c}}=\lambda_{\mathrm{de} \text { Broglie }}^{2}(R / a)^{2} v_{\mathrm{e}}(h / \Delta E)
$$

establishes that the degeneracy factor is much less than one and that each Fermionic electron is independent, without the need of the statistics commonly used for Bosonic photons. The volume is determined by the values of longitudinal and transverse coherences; $V_{\mathrm{c}}$ is on the order of $10^{6} \mathrm{~nm}^{3}$ for typical values of $R$ (distance to the source), $a$ (source dimension), $v_{\mathrm{e}}$ (electron velocity), and $\Delta E$ (energy spread). Unlike the situation in transmission electron microscopy (TEM), coherence and image resolution in UEM are thus determined by properties of the optical field, the ability to focus electrons on the ultrashort time scale, and the operational current density. For both "single electron" and "single pulse" modes of UEM, these are important considerations for achieving the ultimate spatiotemporal resolutions for studies of materials and biological systems, and the question becomes: with ultrashort packets of electrons, can we achieve atomic-scale spatial resolution in images?

In this letter, we report our first atomic-scale resolution in real-space imaging achieved in Caltech's second-generation ultrafast electron microscopy (UEM2) laboratory. With UEM2, which operates at $200 \mathrm{keV}\left(\lambda_{\text {de Broglie }}=2.5079 \mathrm{pm}\right)$, we also report energy-space (electron energy loss spectroscopy, EELS) and Fourier-space (diffraction) patterns of nanostructured materials. The apparatus can operate in the scanning transmission electron microscope (STEM) mode and is designed to explore the vast parameter space bridging the gap between the two ideal operating modes of singleelectron and single-pulse imaging. With these features, UEM studies provide new limits of resolution, image mapping, and elemental analysis. Here, we demonstrate the potential by studying gold particles and islands, boron nitride crystallites, and organometallic phthalocyanine crystals.

Figure 1 displays the conceptual design of UEM2, which, as with the first generation (UEM1), consists of a femtosecond laser system and an electron microscope modified for pulsed operation with femtosecond electron packets. The laser in this case is a diode-pumped $\mathrm{Yb}$-doped fiber oscillator/ amplifier (Clark-MXR; in development), which produces ultrashort pulses of up to $10 \mu \mathrm{J}$ at $1030 \mathrm{~nm}$ with variable pulse width (200 fs to $10 \mathrm{ps)}$ and repetition rate $(200 \mathrm{kHz}$ to $25 \mathrm{MHz}$ ). The output pulses pass through two successive nonlinear crystals to be frequency doubled $(515 \mathrm{~nm})$ and tripled $(343 \mathrm{~nm})$. The harmonics are separated from the residual infrared radiation (IR) beam by dichroic mirrors, and the frequency-tripled pulses are introduced to the photocathode of the microscope for generating the electron pulse train. The residual IR fundamental and frequencydoubled beams remain available to heat or excite samples and clock the time through a computer-controlled optical delay line for time-resolved applications.

The electron microscope column is that of a designed hybrid $200 \mathrm{kV}$ TEM (Tecnai 20, FEI) integrated with two ports for optical access, one leading to the photocathode and the other to the specimen. ${ }^{15}$ The field emission gun (FEG) in the electron-generation assembly adapts a lanthanum hexa- boride $\left(\mathrm{LaB}_{6}\right)$ filament as the cathode, terminating in a conical electron source truncated to leave a flat tip area with a diameter of $16 \mu \mathrm{m}$. The tip is located in a field environment controlled by suppressor and extractor electrodes. The gun can be operated as either a thermal emission or a photoemission source.

The optical pulses are guided to the photocathode as well as to the specimen by a computer-controlled, fine-steering mirror in an externally mounted and X-ray-shielded periscope assembly. Each laser beam can be focused to a spot size of $<30 \mu \mathrm{m}$ full width at half-maximum (fwhm) at its respective target when the beam is expanded to utilize the available acceptance angle of the optical path. Various pulse-energy, pulse-length, and focusing regimes have been used in the measurements reported here. For all UEM measurements, the cathode was heated to a level below that needed to produce detectable thermal emission, as detailed below, and we obtained images using both the TEM and the UEM mode of operation.

For applications involving EELS and energy filtered transmission electron microscopy (EFTEM), we used the Gatan imaging filter (GIF) Tridiem, of the so-called postcolumn type, attached below the camera chamber. The GIF accepts electrons passing through an entrance aperture in the center of the projection chamber. The electron beam passes through a $90^{\circ}$ sector magnet, as shown in Figure 1, which bends the primary beam through a $10 \mathrm{~cm}$ bending radius and thereby separates the electrons according to their energy into an energy spectrum. An energy resolution of $0.87 \mathrm{eV}$ was measured for the EELS zero-loss peak in thermal mode operation of the TEM. A retractable slit is located after the magnet followed by a series of lenses. The lenses restore the image or diffraction pattern at the entrance aperture, and finally it can be recorded on a charge-coupled device (CCD) camera (UltraScan 1000 FT) at the end of the GIF with the Digital Micrograph software. The digital camera uses a $2048 \times 2048$ pixel CCD chip with $14 \mu \mathrm{m}$ square pixels. Readout of the CCD is done as four independent quadrants via four separate digitizing signal chains. This 4-port readout camera combines single-electron sensitivity and 16-bit pixel depth with high-speed sensor readout (4 Mpix/s).

Additionally, for scanning transmission electron microscopy (STEM), the UEM is equipped with a bright-field (BF) detector with a diameter of $7 \mathrm{~mm}$ and an annular dark-field (ADF) detector with an inner diameter of $7 \mathrm{~mm}$ and an outer diameter of $20 \mathrm{~mm}$. Both detectors are located in the nearaxis position underneath the projection chamber. The BF detector usually collects the same signal as the TEM BF image, i.e., the transmitted electrons, while the ADF detector collects an annulus at higher angle where only scattered electrons are detected. The STEM images are recorded with the Tecnai Imaging and Analysis (TIA) software.

To observe the diffraction pattern, i.e., the back focal plane of the objective lens, we inserted a selected area aperture into the image plane of the objective lens, thus creating a virtual aperture in the plane of the specimen. The result is a selected area diffraction (SAD) pattern of the region of interest only. Adjustment of the intermediate and projector 


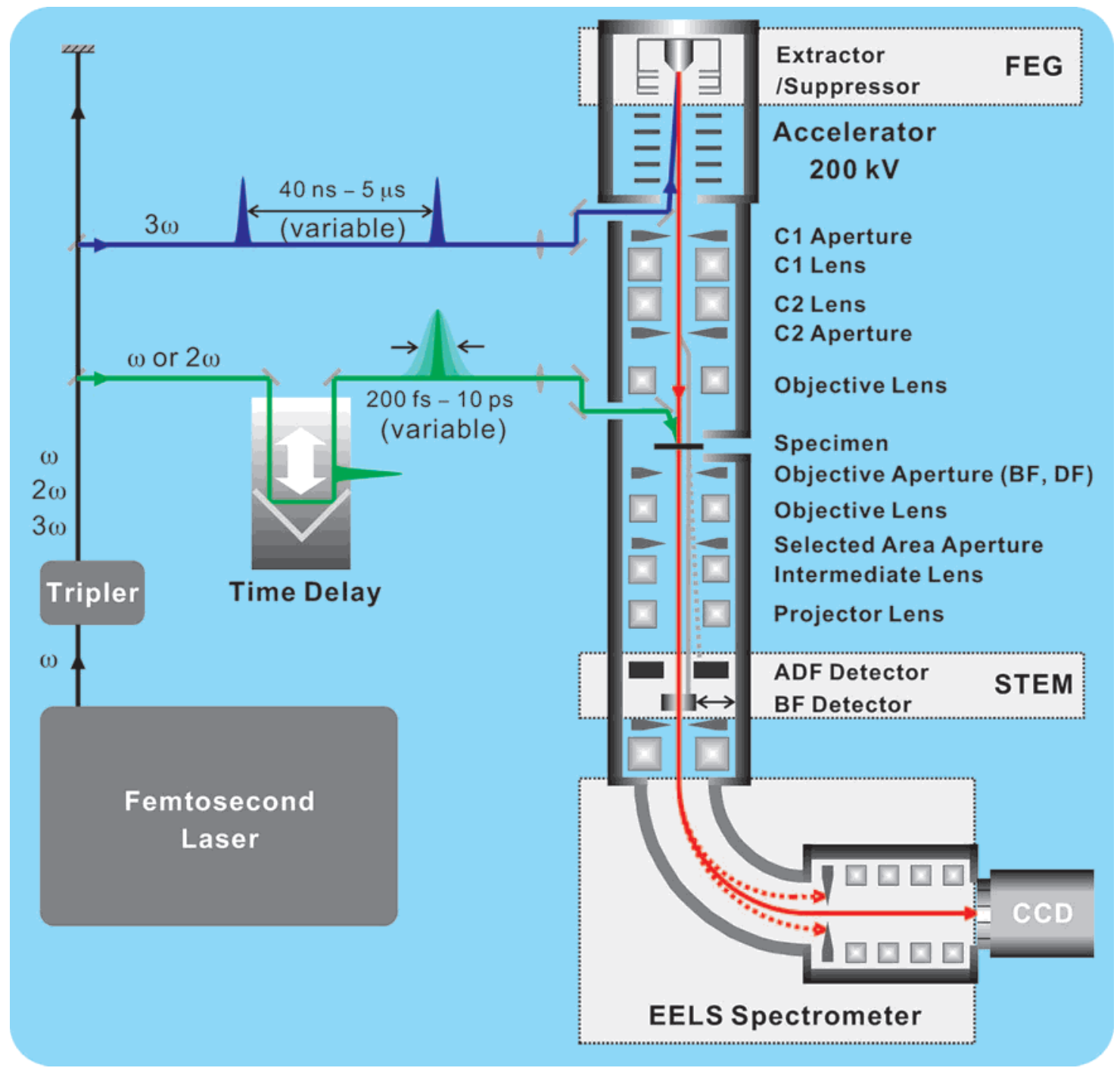

Figure 1. Conceptual design of Caltech's UEM2. A schematic representation of optical, electric, and magnetic components are displayed. The optical pulse train generated from the laser, in this case having a variable pulse width of 200 fs to 10 ps and a variable repetition rate of $200 \mathrm{kHz}$ to $25 \mathrm{MHz}$, is divided into two parts, after harmonic generation, and guided toward the entries of the designed hybrid electron microscope. The frequency-tripled optical pulses are converted to the corresponding probe electron pulses at the photocathode in the hybrid FEG, whereas the other optical pump beam excites (T-jump or electronic excitation) the specimen with a well-defined time delay with respect to the probe electron beam. The probe electron beam through the specimen can be recorded as an image (normal or filtered, EFTEM), a diffraction pattern, or an EEL spectrum. The STEM bright-field detector is retractable when it is not in use. See the text for details.

lens determines the camera length. Diffraction patterns are processed and analyzed for crystal structure determination.

Several key features realized in the new UEM2 machine are worth emphasizing. First, the high repetition rate amplified laser source allows us to illuminate the cathode with $343 \mathrm{~nm}$ pulses of energies above $500 \mathrm{~nJ}$ compared with typical values of $3 \mathrm{~nJ}$ near $380 \mathrm{~nm}$ for UEM1. Thus, we are able to deliver a level of average optical power for electron generation comparable to that of UEM1 operating at $80 \mathrm{MHz}$, but at much lower repetition rates. The pulse energy available in the visible and IR beams is also at least 2 orders of magnitude greater than for UEM1, allowing for exploration of a much greater range in the choice of sample excitation conditions.

Second, the hybrid $200 \mathrm{kV}$ FEG, incorporating an extractor/suppressor assembly providing an extractor potential of up to $4 \mathrm{kV}$, allows higher resolving power and greater flexibility and control of the conditions of electron generation. Third, with simple variation of optical pulse width, we can control the temporal and spatial resolution depending on the requirements of each experiment. Fourth, with variation of spacing between optical pulses without loss of pulse energy, we can explore a wide range of samples, allowing them to fully relax their energy after each excitation pulse and rewind the clock precisely; with enough electrons, below the space-charge limit, single-pulse recording is possible. Finally, by the integration of the EELS spectrometer, our new machine is empowered with energy resolution in addition to the ultrafast time resolution and atomic-scale space resolution.

In what follows, we present results demonstrating the capabilities of UEM2 in three areas: real-space imaging, diffraction, and electron energy resolution. We first discuss the images recorded in the UEM mode, of gold particles and 

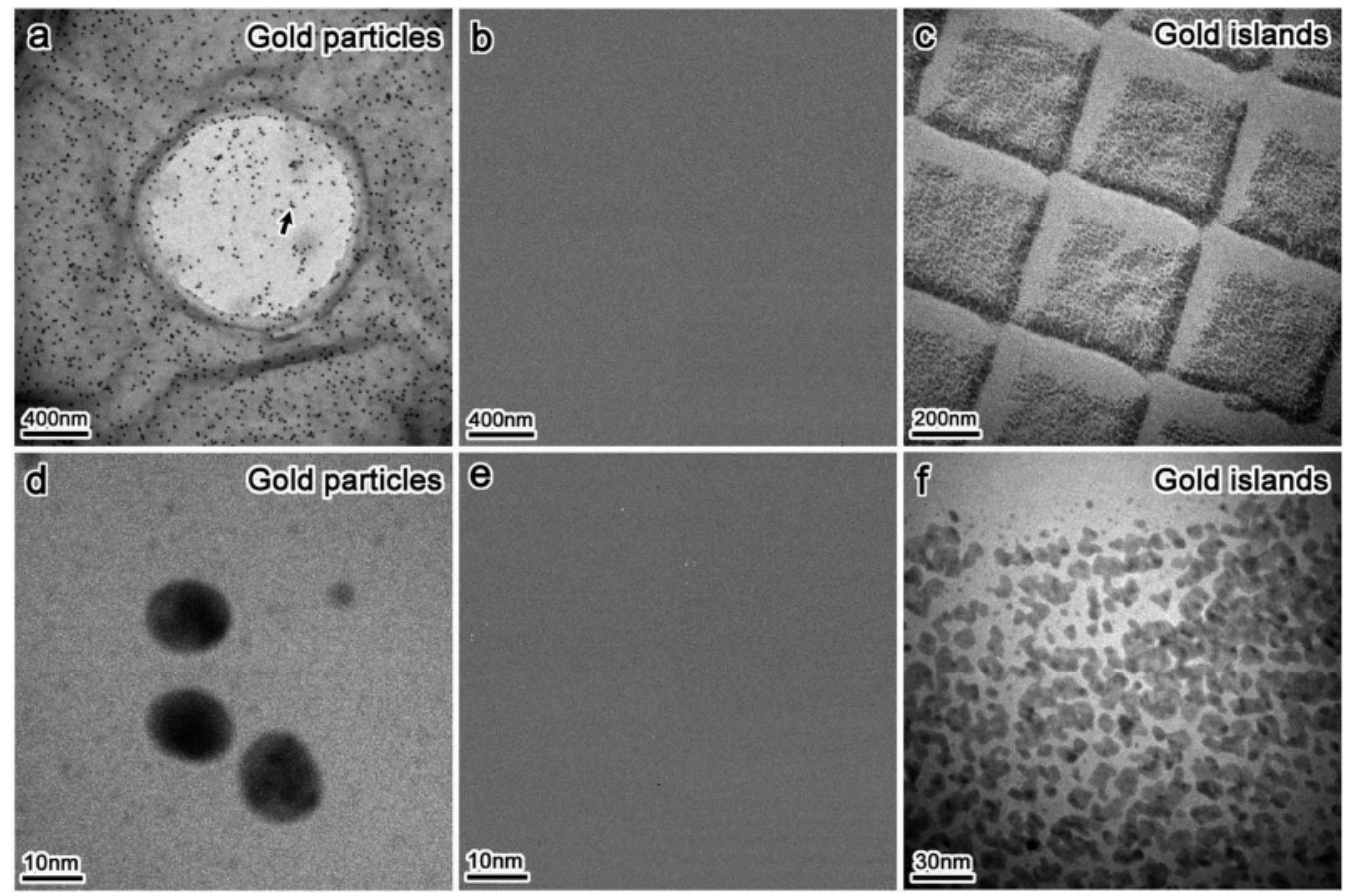

Figure 2. UEM images obtained with ultrafast electron pulses. Shown are gold particles (a,d) and gold islands (c,f) on carbon films. UEM background images $(\mathrm{b}, \mathrm{e})$ obtained by blocking the photoelectron-extracting femtosecond laser pulses. For the UEM images of gold particles, we used the objective (contrast) aperture of $40 \mu \mathrm{m}$ to eliminate diffracted beams, while no objective aperture was used for the gold-island images.
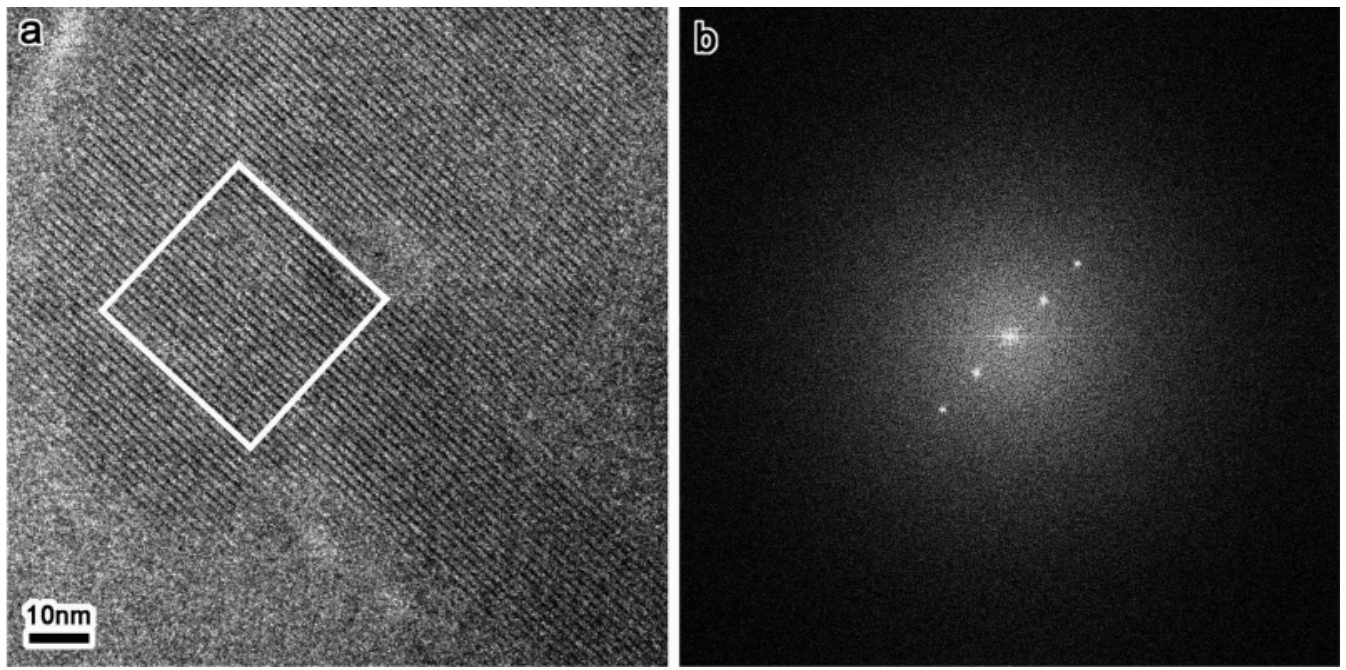

Figure 3. High-resolution, phase-contrast UEM images. Shown are the image (a) and digital diffractogram (b) of an organometallic crystal of chlorinated copper phthalocyanine. The diffractogram was obtained by the Fourier transform of the image in (a). The high-resolution image was taken near the Scherzer focus for optimum contrast, which was calculated to be $90.36 \mathrm{~nm}$ for a spherical aberration coefficient $C$ s of the objective lens of $2.26 \mathrm{~mm}$. The objective aperture was not used.

gold islands on carbon films. Panels a and d of Figure 2 show gold particles of uniform size dispersed on a carbon film. From the higher magnification image of Figure 2d, corresponding to the area indicated by the black arrow in Figure 2a, it is found that the gold particles have a size of $15 \mathrm{~nm}$, and the minimum particle separation seen in the image is $3 \mathrm{~nm}$. It should be noted that panels $b$ and e of Figure 2 were recorded under identical conditions to panels a and $\mathrm{d}$ of Figure 2, respectively, but without cathode irradiation by the femtosecond laser pulses. No images were observed, demonstrating that nonoptically generated electrons from our warm cathode were negligible. Similar background images with the light pulses blocked were routinely recorded and checked for all cathode conditions used in this study. The waffle (cross line) spacing of the cross-grating replica (gold islands) seen in Figure 2c is known to be $463 \mathrm{~nm}$. The gold islands are observed in Figure 2f, where the bright regions correspond to the amorphous carbon support film and the dark regions to the nanocrystalline gold islands. It is found that the islands may be interconnected or isolated depending on the volume fraction of the nanocrystalline phases.

To test the high-resolution capability of UEM by means of phase contrast imaging, we investigated an organometallic 

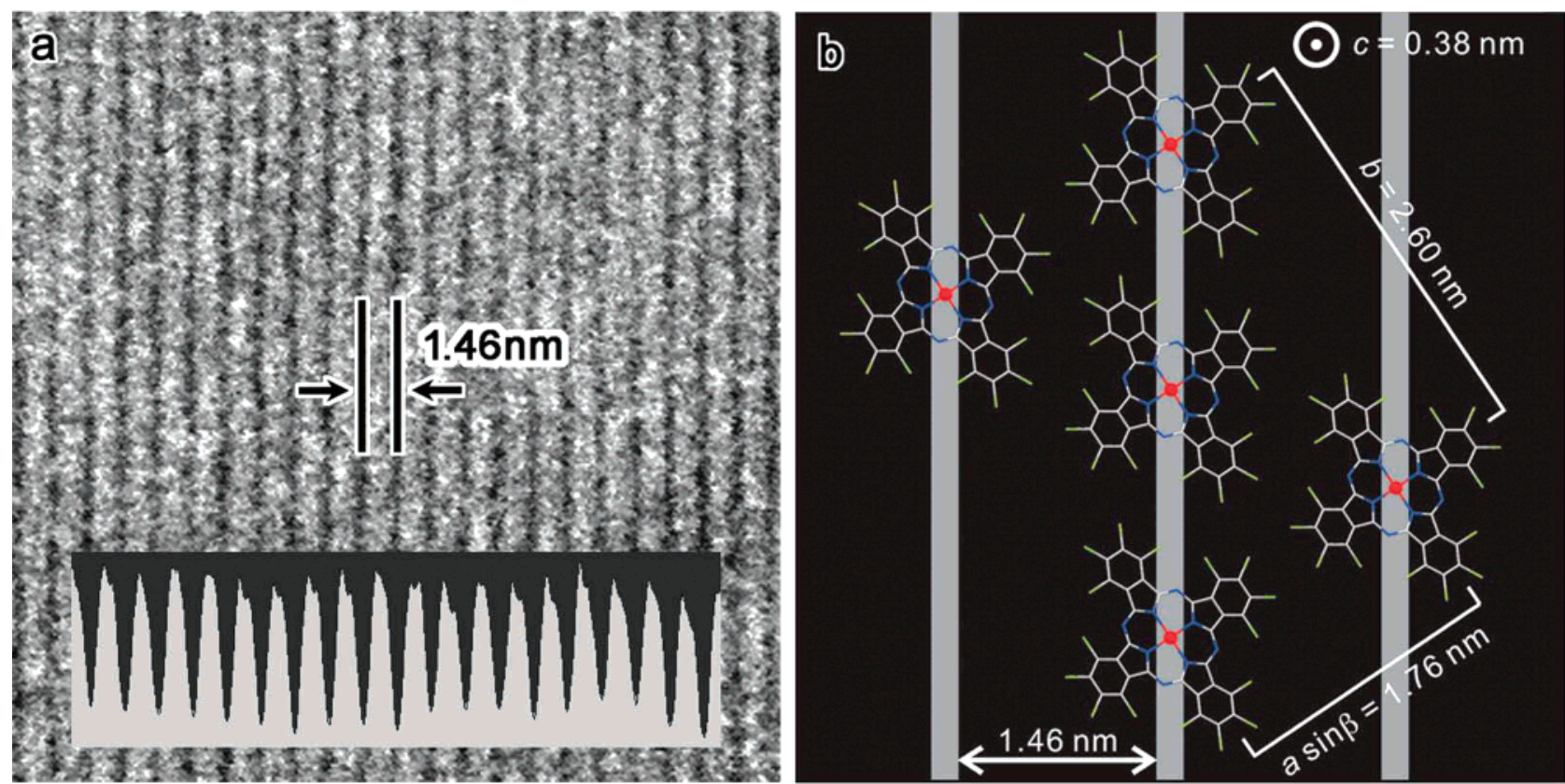

Figure 4. High-resolution, phase-contrast UEM image and structure of chlorinated copper phthalocyanine. The high-resolution image (a) is a magnified view of the outlined area in Figure 3a. The representation of the crystal structure (b) is shown in projection along the $c$-axis, ${ }^{17}$ and the assignment of the copper planes observed in (a) is indicated by the gray lines. The red spheres are the copper atoms.
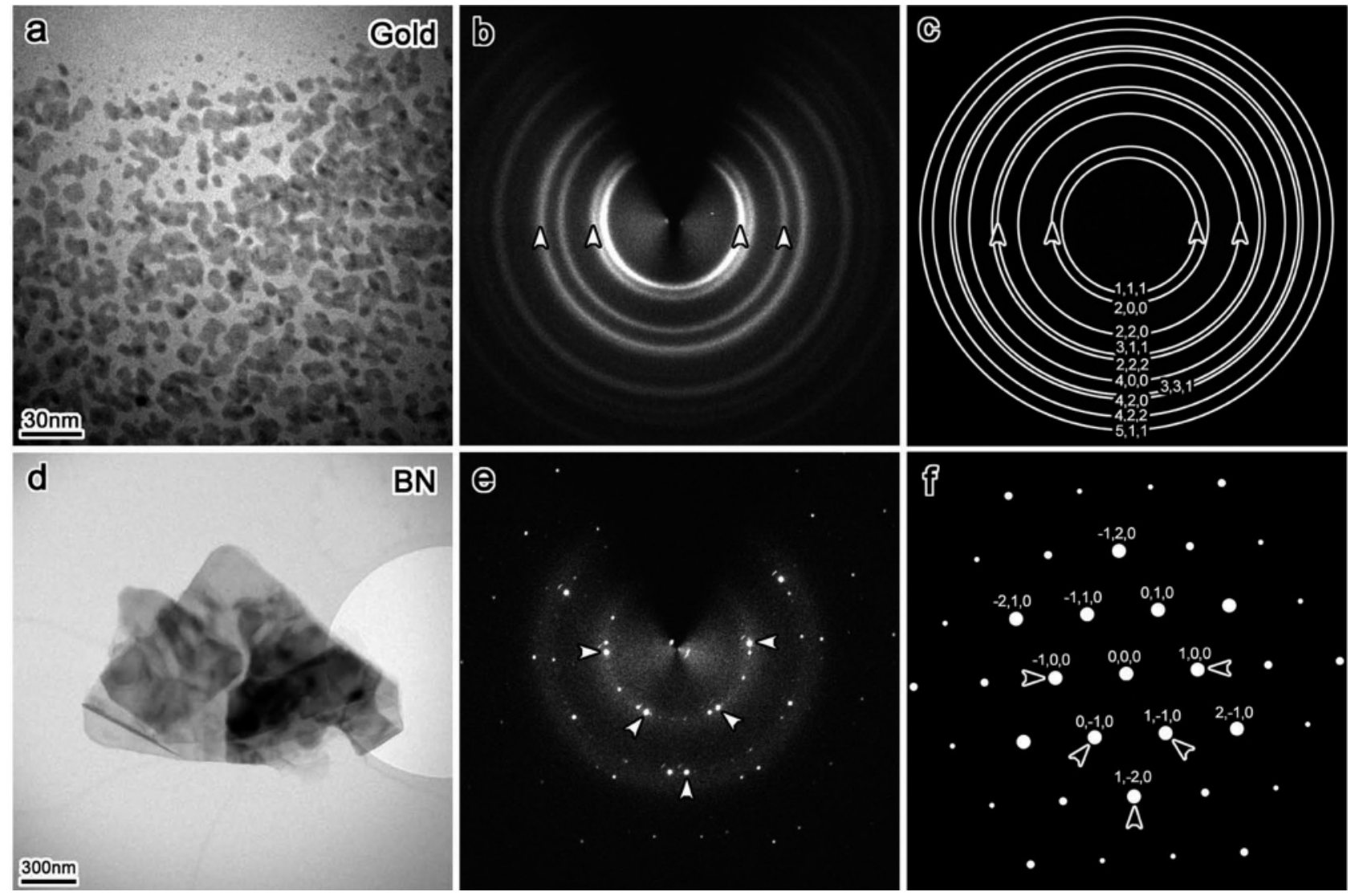

Figure 5. UEM images and electron diffraction patterns. Shown are images and measured and calculated electron diffraction patterns of gold islands $(\mathrm{a}-\mathrm{c})$ and boron nitride $(\mathrm{BN})(\mathrm{d}-\mathrm{f})$ on carbon films. The incident electron beam is parallel to the [001] direction of the BN. All diffraction patterns were obtained by using the selected-area diffraction (SAD) aperture, which selected an area $6 \mu \mathrm{m}$ in diameter on the specimen. Representative diffraction spots and rings were indexed as indicated by the arrowheads.

compound, chlorinated copper phthalocyanine (hexadecachlorophthalocyanine, $\left.\mathrm{C}_{32} \mathrm{Cl}_{16} \mathrm{CuN}_{8}\right) .{ }^{16}$ The major spacings of lattice fringes of copper of this molecule in projection along the $c$-axis are known to be $0.88,1.30$, and $1.46 \mathrm{~nm}$, with atomic spacings of 1.57 and $1.76 \mathrm{~nm} .{ }^{17}$ Figure $3 \mathrm{a}$ exhibits the lattice fringes observed by UEM, where the black 


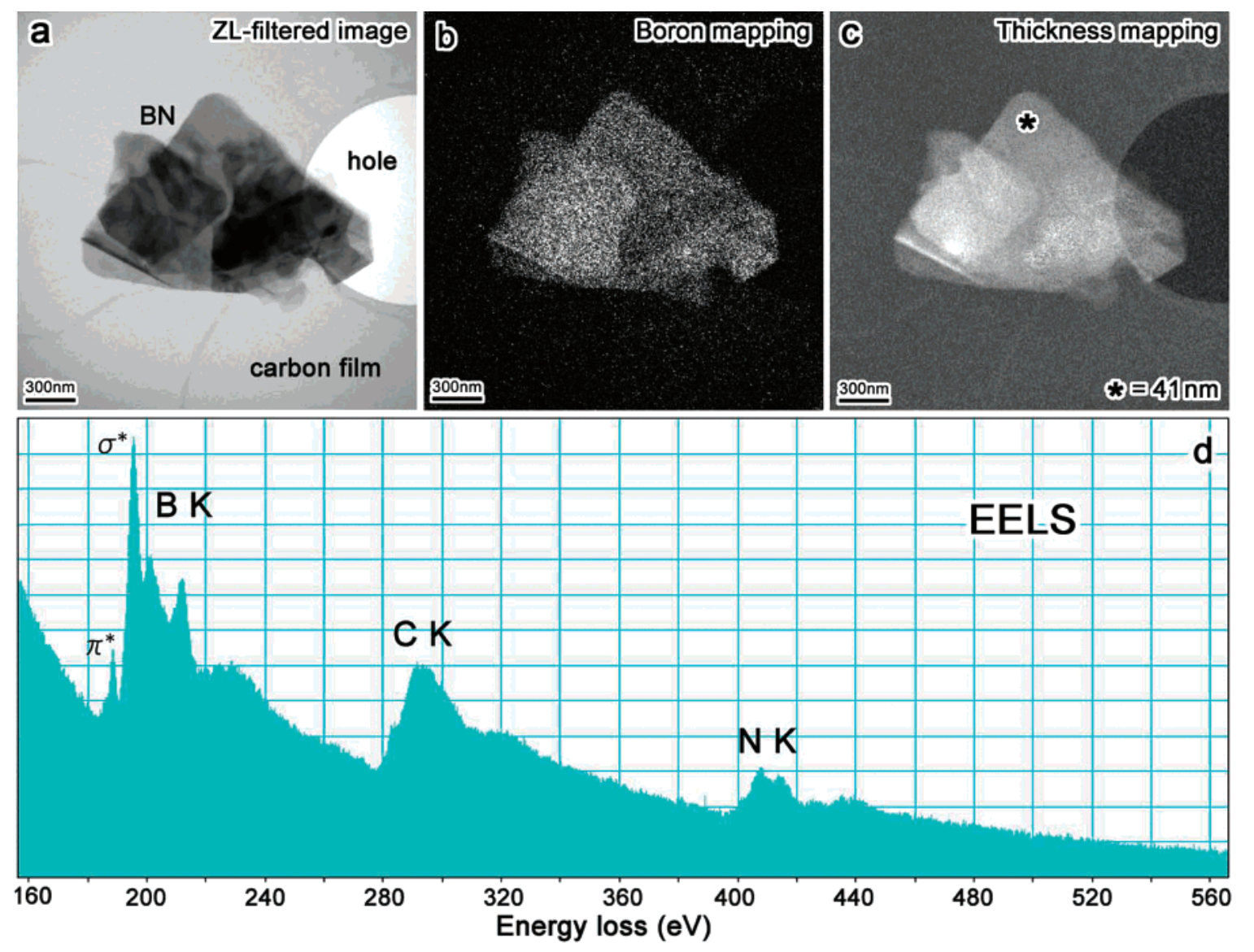

Figure 6. Energy-filtered UEM images and spectrum. A zero-loss filtered image (a), boron K-edge mapping image (b), thickness mapping image (c), and electron energy loss (EEL) spectrum (d) of the boron nitride (BN) sample. We used the 5.0 and $1.0 \mathrm{~mm}$ entrance aperture for mapping images and EEL spectrum, respectively. The thickness at the point indicated by the asterisk in (b) is estimated as $41 \mathrm{~nm}$. ZL stands for zero-loss.

lines correspond to copper layers parallel to the $c$-axis. The Fourier transform of Figure $3 \mathrm{a}$ is shown in Figure 3b, discussed below, and the clear reciprocity (without satellite peaks in the F.T.) indicates the high degree of order in crystal structure.

Figure $4 \mathrm{a}$ is an enlargement of the area outlined in Figure $3 \mathrm{a}$, clearly showing the lattice fringe spacing of 1.46 $\mathrm{nm}$, corresponding to the copper planes highlighted in gray in Figure $4 \mathrm{~b}$ in which a unit cell is shown in projection along the $c$-axis. It is known that, in high-resolution images, the lattice fringes produced by the interference of two waves passing through the back focal plane, i.e., the transmitted and diffracted beams, are observed only in crystals where the lattice spacing is larger than the resolution of the TEM. ${ }^{18}$ In the profile inset of Figure 4a, it should be noted that the fwhm was measured to be less than $7 \AA$, directly indicating that our UEM has the capability of subnanometer resolution.

The digital diffractogram obtained by the Fourier transform of the observed high-resolution image of Figure $3 \mathrm{a}$ is shown in Figure 3b. In the digital diffractogram, the peaks represent the fundamental spatial frequency of the copper layers $\left(0.69 \mathrm{~nm}^{-1}\right)$ and higher harmonics thereof. A more powerful means of obtaining reciprocal-space information such as this is the direct recording of electron diffraction, also available in UEM. Figure 5 shows measured and calculated electron diffraction patterns of gold islands and boron nitride $(\mathrm{BN})$ on carbon films, along with the corresponding real-space images of each specimen, all recorded by UEM. In Figure $5 b$, the electron diffraction patterns exhibit DebyeScherrer rings formed by numerous diffraction spots from a large number of face-centered gold nanocrystals with random orientations. The rings can be indexed as indicated by the white arrowheads. The diffraction pattern of $\mathrm{BN}$ in Figure $5 \mathrm{e}$ is indexed by the hexagonal structure projected along the [001] axis, as shown in Figure 5f. It can be seen that there are several BN crystals with different crystal orientations besides that responsible for the main diffraction spots indicated by the white arrowheads.

To explore the energy resolution of UEM, we investigated the BN specimen in detail by EELS and EFTEM. Figure 6 shows a zero-loss filtered image (a), boron K-edge mapping image (b), thickness mapping image (c), and the corresponding EEL spectrum (d). The boron map was obtained by the so-called three-window method. ${ }^{19}$ In the boron map of Figure $6 \mathrm{~b}$, image intensity is directly related to areal intensity of boron. In the thickness map of Figure $6 \mathrm{c}$, the brightness increases with increasing thickness: $d=\lambda(\beta) \ln \left(I_{t} / I_{0}\right)$, where $\lambda$ is the mean free path for inelastic scattering for a given collection angle $\beta, I_{0}$ is the zero-loss (ZL) peak intensity, and $I_{\mathrm{t}}$ is the total intensity. The thickness in the region 
indicated by the asterisk in Figure 6c was estimated to be $41 \mathrm{~nm}$. In the EEL spectrum of Figure 6d, the boron K-edge, carbon K-edge, and nitrogen K-edge are observed at the energy of 188, 284, and $401 \mathrm{eV}$, respectively. In the boron K-edge spectrum, sharp $\pi^{*}$ and $\sigma^{*}$ peaks are clearly visible. The carbon K-edge spectrum is considered to result from the amorphous carbon film due to the existence of small and broad peaks at the position $\pi^{*}$ and $\sigma^{*}$, being quite different from spectra of diamond and graphite.

With the new capabilities of UEM2, we can now study structural dynamics, as with UEM1, ${ }^{1,9,11}$ but with the new energy and spatial resolution achieved here. Specimens will be excited in a T-jump or electronic excitation by the femtosecond laser pulses (Figure 1) scanned in time with respect to the electron packets, which will probe the changes induced in material properties through diffraction, imaging, or electron energy loss in different energy regions, including that of Compton scattering. We also plan to explore the STEM feature in UEM, particularly the annular dark-field imaging, in which compositional changes are evident in the contrast ( $Z$ contrast). Such images are known to offer advantages over high-resolution TEM (relative insensitivity to focusing errors and ease of interpretation $\left.{ }^{20}\right)$. Electron fluxes will be optimized either through changes of the impinging pulse fluence or by designing new photocathode materials. In this regard, with higher brightness, we should be able to reach the subangstrom regime at $300 \mathrm{keV}$. The potential for applications in materials and biological research is evidently rich.

Acknowledgment. This work was supported by the Gordon and Betty Moore Foundation, the National Science Foundation, and the Air Force Office of Scientific Research. We thank Mike Stekelenburg and Bill Anderson for their efforts during the design and installation of the UEM2 microscope and Tony Carpenter for his timely support. We are also grateful to Dr. Vladimir Lobastov for helpful discussions, Dr. William Tivol for his interest and supply of some TEM samples, and Prof. Eberhard Riedle and Dr. Peter Baum for providing a specially designed tripling crystal.

\section{References}

(1) Zewail, A. H. Annu. Rev. Phys. Chem. 2006, 57, 65-103 and references therein

(2) Ihee, H.; Lobastov, V. A.; Gomez, U. M.; Goodson, B. M.; Srinivasan, R.; Ruan, C.-Y.; Zewail, A. H. Science 2001, 291, 458462.

(3) Srinivasan, R.; Lobastov, V. A.; Ruan, C.-Y.; Zewail, A. H. Helv. Chim. Acta 2003, 86, 1761-1838 and references therein.

(4) Srinivasan, R.; Feenstra, J. S.; Park, S. T.; Xu, S.; Zewail, A. H. Science 2005, 307, 558-563.

(5) Ruan, C.-Y.; Lobastov, V. A.; Vigliotti, F.; Chen, S.; Zewail, A. H. Science 2004, 304, 80-84.

(6) Thomas, J. M. Angew. Chem., Int. Ed. 2004, 43, 2606-2610.

(7) Thomas, J. M. Angew. Chem., Int. Ed. 2005, 44, 5563-5566.

(8) Lobastov, V. A.; Srinivasan, R.; Zewail, A. H. Proc. Natl. Acad. Sci. U.S.A. 2005, 102, 7069-7073.

(9) Grinolds, M. S.; Lobastov, V. A.; Weissenrieder, J.; Zewail, A. H. Proc. Natl. Acad. Sci. U.S.A. 2006, 103, 18427-18431.

(10) Gedik, N.; Yang, D.-S.; Logvenov, G.; Bozovic, I.; Zewail, A. H. Science 2007, 316, 425-429.

(11) Lobastov, V. A.; Weissenrieder, J.; Tang, J.; Zewail, A. H. Nano Lett. 2007, 7, 2552-2558.

(12) (a) Yang, D.-S.; Gedik, N.; Zewail, A. H. J. Phys. Chem. C 2007, 111, 4889-4919. (b) Seidel, M. T.; Chen, S.; Zewail, A. H. J. Phys. Chem. C 2007, 111, 4920-4938.

(13) Baum, P.; Zewail, A. H. Proc. Natl. Acad. Sci. U.S.A. 2006, 103, 16105-16110.

(14) Zewail, A. H. In Visions of Discovery: Shedding New Light on Physics and Cosmology; Chiao, R., Phillips, W., Leggett, A., Cohen, M., Ellis, G., York, D., Bishop, R., Harper, C., Eds.; Cambridge University Press: Cambridge, in press.

(15) Zewail, A. H.; Lobastov, V. Method and System for Ultrafast Photoelectron Microscope. U.S. Patent 7,154,091 B2, 20050401, 2006.

(16) Murata, Y.; Baird, T.; Fryer, J. R. Nature 1976, 262, 721-722.

(17) Murata, Y.; Fryer, J. R.; Baird, T.; Murata, H. Acta Crystallogr. 1977, A33, 198-200

(18) Shindo, D.; Hiraga, K. High-Resolution Electron Microscopy for Materials Science; Springer-Verlag: Tokyo, 1998.

(19) Brydson, R. Electron Energy Loss Spectroscopy; BIOS: Oxford, 2001

(20) Science of Microscopy; Hawkes, P. W., Spence, J. C. H., Eds.; Springer: New York, 2007 and references therein.

NL071369Q 